\title{
¿Qué avances recientes hay en el entendimiento, diagnóstico y tratamiento de la enfermedad de Von Willebrand?: una revisión de la literatura
}

What Recent Advances are there in the Understanding, Diagnosis and Treatment of von Willebrand Disease? A Literature Review

\author{
Catherin Tovar SÁnchez \\ Médica cirujano, Facultad de Medicina, Pontificia \\ Universidad Javeriana, Bogotá, Colombia \\ Alexander Salazar Reviakina \\ Estudiante de Medicina, Pontificia Universidad \\ Javeriana, Bogotá, Colombia \\ José Alejandro Rumbo Romero \\ Estudiante de Medicina, Pontificia Universidad \\ Javeriana, Bogotá, Colombia \\ María Manuela Sierra Bretón \\ Estudiante de Medicina, Pontificia Universidad \\ Javeriana, Bogotá, Colombia \\ Ithzayana Madariaga Perpiñán \\ Médico cirujano, Facultad de Medicina, Pontificia \\ Universidad Javeriana, Bogotá, Colombia \\ Ignacio Zarante Montoya \\ Médico genetista. Profesor del Instituto de Genética \\ Humana, Facultad de Medicina, Pontificia Universidad \\ Javeriana, Bogotá, Colombia
}

$\begin{array}{lll}\text { a Autora de correspondencia: } & \text { de } \\ \text { catherin.tovar@javeriana.edu.co } & \end{array}$

Cómo citar: Tovar Sánchez C, Salazar-Reviakina A, Rumbo Romero JA, Sierra Bretón MM, Madariaga Perpiñán I, Zarante Montoya I. ¿Qué avances recientes hay en el entendimiento, diagnóstico y tratamiento de la enfermedad de Von Willebrand?: una revisión de la literatura. Univ. Med. 2020;61(2). https://doi.org/10.11144/Javeriana.umed61-2.vonw

\section{RESUMEN}

La enfermedad de Von Willebrand (EVW) es el trastorno hemorrágico hereditario más común. En los últimos años han ocurrido grandes avances en su entendimiento, diagnóstico y tratamiento. Afecta hasta el $1 \%$ de la población y comprende un espectro de subtipos heterogéneos. Se caracteriza por mutaciones con una disminución en el nivel o deterioro en la acción del factor de Von Willebrand (FVW). La mayoría de los casos se transmiten como un rasgo autosómico dominante. Las pruebas de diagnóstico para este trastorno son complejas y su interpretación requiere una comprensión de la fisiopatología. El arsenal terapéutico disponible incluye el uso de desmopresina y los concentrados de FVW/FVIII en procedimientos que requieran hemostasia. La aplicación rutinaria de terapia profiláctica para eventos hemorrágicos no se encuentra indicada. El objetivo de esta revisión es discutir la epidemiología, fisiopatología y los más recientes avances en el diagnóstico y tratamiento de la EVW hereditaria.

Palabras clave

enfermedad de Von Willebrand; factor de Von Willebrand; trastornos hemorrágicos; desamino arginina vasopresina. 


\begin{abstract}
Von Willebrand disease (vWD) is the most common hereditary bleeding disorder, affects up to $1 \%$ of the population and comprises a spectrum of heterogeneous subtypes. It is characterized by mutations that lead to a decrease in the level or deterioration in the action of von Willebrand factor (vWF). Most cases are transmitted as autosomal dominant. The diagnostic tests for this disorder are complex, and the proper interpretation requires a thorough understanding of the underlying pathophysiology by the health care group. The objective of this review is to discuss the epidemiology, pathophysiology, and the most recent advances in the diagnosis and treatment of $\mathrm{vWD}$.

Keywords

von Willebrand disease; von Willebrand factor; hemorrhagic diathesis; deamino arginine vasopressin.
\end{abstract}

\section{Introducción}

La enfermedad de Von Willebrand (EVW) es un trastorno hereditario que cursa con hemorragias de intensidad variable, causado por alteraciones cuantitativas, cualitativas o funcionales del factor de Von Willebrand (FVW) (1). El FVW es esencial para la unión de las plaquetas al subendotelio de vasos lesionados y para el transporte del factor VIII (FVIII) (2).

La EVW es el trastorno hemorrágico hereditario más común, pues afecta aproximadamente al $1 \%$ de la población (3). Se estima que en Estados Unidos, la prevalencia de esta enfermedad es del $1 \%$ y en Colombia, hasta 2018, representaba la tercera enfermedad huérfana más frecuente con una tasa de 1,4 individuos por cada 100.000 habitantes (4). La mayoría de los casos se transmiten como un rasgo autosómico dominante sin predilección por ningún sexo (5).

A continuación, se hará una revisión general de la EVW hereditaria, con información actualizada acerca de su fisiopatología, diagnóstico y tratamiento, con el objetivo de facilitar su abordaje clínico y paraclínico para el médico de atención primaria.

\section{Metodología}

Se realizó una revisión narrativa de la literatura. Se incluyeron las siguientes bases de datos electrónicas en las búsquedas bibliográficas: Medline vía PubMed, ScienceDirect, Clinical Key, Scopus, EBSCO y SciELO. Se realizaron búsquedas con uso de términos $\mathrm{MeSH}$ (Medical Subject Headings) y DeCS (Descriptores en Ciencias de la Salud) dependiendo del idioma de publicación de los artículos revisados (inglés y español). Los términos utilizados fueron: enfermedad de Von Willebrand, factor de Von Willebrand, trastornos hemorrágicos, desmopresina. Adicionalmente, se revisaron libros de texto y guías de práctica clínica publicadas por sociedades científicas en el mundo y colombianas, como el Fondo Colombiano de la Hemofilia y el Fondo Nacional de Enfermedades de Alto Costo. Dentro de la revisión se tomaron artículos desde 1987 hasta el 2019, con excepción de la sección de tratamiento, en la que se consideraron únicamente artículos publicados desde 2012 hasta 2019.

\section{Historia}

La primera descripción la realizó Erik Von Willebrand, en 1926, quien inicialmente consideró un problema de sangrado mucocutáneo severo presente en una familia del mar Báltico, en la cual varios integrantes fallecieron debido a hemorragias graves de múltiples orígenes (6).

Durante los años cincuenta se descubrió una relación con la disminución de la actividad del FVIII, que lograba ser corregida con plasma de pacientes sanos. Durante la década de los sesenta, el noruego Christian Borchgrevink vislumbró los primeros indicios del rol del FVW en la agregación plaquetaria, información confirmada en 1970 por Margaret Howard y Barry Firkin a través de sus estudios con ristocetina, un antibiótico que induce el proceso de agregación en individuos sanos y hemofílicos, pero no en pacientes con EVW (7). Finalmente, hacia 1985 el gen del FVW es aislado y en 1989 se determina su estructura, gracias a lo cual se confirma que la EVW y la hemofilia A son entidades distintas codificadas por genes independientes (6). 


\section{Epidemiología}

La EVW es la patologi\#a hemorra\#gica hereditaria autoso\#mica ma\#s frecuente. Su prevalencia ha sido estimada en un $1 \%(8,9)$. En población caucásica se estima una frecuencia desde 30 casos por millo\#n de habitantes hasta el $1 \%$ de la poblacio\#n (10). Los casos sintomáticos se estiman en 100 por millo\#n de habitantes. Se estiman 620.000 casos sintomáticos en el mundo, de los cuales aproximadamente el $80 \%$ habita en países en vía de desarrollo (11).

Aproximadamente, 125 individuos por millo\#n presentan EVW sintomática en los Estados Unidos. En un estudio realizado en el norte de Italia informaron una prevalencia del $0,9 \%$, aproximadamente 8,2 casos por cada 1000 habitantes (12).

Hay tres tipos principales de EVW. El tipo 1 se debe a una reducción cuantitativa en la proteína del FVW; el tipo 2, a un FVW disfuncional, y el tipo 3, a FVW ausente o muy reducido. De los pacientes con EVW, el 70-80\% son tipo 1 , el $5-15 \%$ son tipo 2 y menos del $5 \%$ son tipo 3. La prevalencia del tipo 3 es de 1 a 5 por millón de habitantes en Europa, y de 3 por millo\#n en Suecia e Israel (13). En Chile y Venezuela representan el 2,71, 1,75 por millón, respectivamente (14).

En Colombia, para el 2018 se reportaron 4271 personas con diagnóstico de coagulopatía, de las cuales el 35,2\% tenía EVW $(15,16)$. En los pacientes con EVW predomina la afectación en el sexo femenino $(74,7 \%)$, con una proporción de casi 2:1, probablemente por el sangrado menstrual excesivo, que facilita su diagnóstico (17). La prevalencia en nuestra población es de 3 por cada 100.000 habitantes, con una media de edad de 28 años $(\mathrm{DE}=16,4)(16)$. Es importante tener en cuenta que no se han encontrado preferencias por raza o zona geográfica en el comportamiento de esta enfermedad (9).

\section{Estructura molecular del factor de Von Willebrand}

El gen para el FVW se localiza en el brazo corto del cromosoma 12, en la región 13.3. Contiene 178 kilobases $(\mathrm{Kb})$ y 52 exones. Existe, además, un seudogen con copia de los exones 23 al 34 en el cromosoma 22. Codifica para ARNm de $8,8 \mathrm{~Kb}$ de longitud, su proteína constituye una glucoproteína plasmática de entre 500 y $2000 \mathrm{KDa}$, posee 2813 aminoácidos, de los cuales 22 equivalen a un péptido señal, 741 a un propolipéptido (dominios D1 y D2) y 2050 a la subunidad del FVW con propiedades adhesivas necesarias para cumplir su función hemostática $(18,19)$. Se han descrito 140 polimorfismos de inserción y deleción ubicados en su región promotora, regiones intrónicas y exones $(6,20,21)$. Se han observado diferentes tipos de mutación para esta patología, como lo son errores transcripcionales o en la remoción de intrones, mutaciones nonsense, missense, deleciones e inserciones (22).

El FVW es sintetizado por células endoteliales y megacariocitos $(20,23)$, almacenado en gránulos alfa y cuerpos de Weibel-Palade (23). Además, es modulado y clivado por las metaloproteinasas ADAMTS 13 al momento de entrar al torrente sanguíneo $(14,20,24)$. La estructura de esta proteína comprende dominios de la $\mathrm{A}$ a la $\mathrm{D}$, estos últimos involucrados en la regulación de la formación multimérica y su adhesión al FVIII de la coagulación. Los dominios A poseen propiedades de fijación al colágeno, y con los dominios $\mathrm{C}$ se encargan de la adhesión plaquetaria.

\section{Fisiopatología}

La EVW se caracteriza por defectos cuantitativos o cualitativos del FVW (25). Este último desempeña una papel fundamental en la localización e inicio de la respuesta hemostática primaria y secundaria $(26,27)$.

Posterior a una lesión endotelial, hay incremento en la concentración local del FVW, por su unión a componentes de la matriz 
subendotelial, como el colágeno. Esta interacción facilita la unión del FVW al receptor del complejo Ib-IX-V de glicoproteína plaquetaria (GPIb-IX-V); adicionalmente, el estrés por cizallamiento produce un cambio en la forma del FVW, facilitando su unión a la glicoproteína Ib plaquetaria (GPIb) (28). Ello permite la adherencia de las plaquetas circulantes al sitio lesionado, al promover su agregación y favorecer la formación del tapón plaquetario (figura 1) (29). La ausencia del FVW o una estructura anormal dificulta el proceso de reclutamiento plaquetario y determina una hemostasia primaria subóptima $(30,31)$.

Figura 1.

Funciones del factor de Von Willebrand

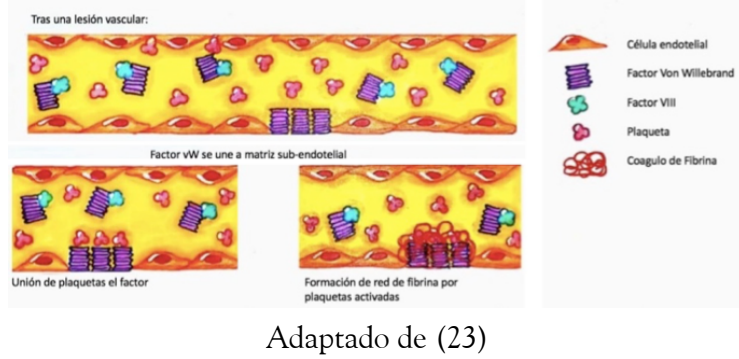

Por otro lado, el FVW es responsable de la protección y transporte del FVIII, pues asegura su contacto con los fosfolípidos de superficie de plaquetas activadas y endotelio lesionado (32). En este sentido, la ausencia del FVW implica una disminución en la vida media del FVIII, lo que genera alteraciones en la hemostasia secundaria (33).

El FVW es el transportador plasmático del factor VIII. Luego de una lesión endotelial, el FVW se une a la matriz subendotelial, y gracias a fuerzas de cizallamiento, sufre un cambio conformacional que permite la adherencia plaquetaria mediante el receptor glicoproteico GPIb. Las plaquetas son activadas e inician el proceso de agregación, alterando sus fosfolípidos de superficie y facilitando la unión del factor VIII. Este último en su interacción con el factor Xa favorece la cascada de coagulación y formación final del coágulo de fibrina.

\section{Clasificación}

La EVW se clasifica en diferentes tipos (tabla 1), que se explican a continuación $(34,35)$.

Tabla 1.

Prevalencia y nivel de deficiencia del factor de Von Willebrand en los tipos de enfermedad de Von Willebrand

\begin{tabular}{|l|l|l|}
\hline Tipo & Prevalencia & Deficiencia del factor \\
\hline Tipo I & $70-80 \%$ & Moderada \\
\hline Tipo IC & $<1 \%$ & Moderada \\
\hline Tipo IIA & $20-25 \%$ & Leve o nula \\
\cline { 3 - 3 } Tipo IIB & & Leve o nula \\
\hline Tipo III & $<5 \%$ & Severa \\
\hline
\end{tabular}

\section{Alteraciones cuantitativas}

Tipo 1. Es la forma más frecuente de la enfermedad. Se caracteriza por una disminución en la cantidad del FVW en la sangre, así como una menor actividad plaquetaria dependiente de este (23). Es heredado de manera autosómica dominante, con mutaciones que afectan la producción en las etapas de empaquetamiento y secreción, lo que lleva a una disminución del FVW sérico útil (21).

Tipo 1C. Es una forma extremadamente rara de la enfermedad, en la cual hay una producción adecuada del factor; sin embargo, se metaboliza de manera ultrarrápida, lo que lleva a una disminución en las concentraciones séricas y a una vida media corta de la proteína (23).

Tipo 3. En esta forma de la enfermedad hay una depleción casi completa o completa del factor, acompañada de deficiencia de FVIII (36). Esto genera formas muy severas de la enfermedad, como sangrados articulares o musculares profundos. Es la forma menos prevalente (37), y la mayoría de pacientes con este tipo de la enfermedad muestran mutaciones recesivas o heterocigotas compuestas (23). 


\section{Alteraciones cualitativas}

Tipo 2A. Esta forma la causa una deficiencia en la formación de FVW funcional, que se da por una disminución o ausencia de multímeros de alto peso molecular o por la formación de multímeros anormales previo a la liberación de la proteína (31). Si bien el problema no se encuentra en la cantidad de FVW, este se puede encontrar disminuido (38).

Tipo 2B. Se caracteriza por una mutación que genera mayor unión entre el dominio AI del FVW y la GPIb-alfa (39). In vivo la unión exagerada de estas dos moléculas aumenta su barrido y lleva a una disminución en la cantidad de FVW. En esta forma de la enfermedad, algunos pacientes cursan con trombocitopenia, lo que se consolida como un factor de riesgo adicional para sangrado (40).

Tipo 2M. Este subtipo de la enfermedad se caracteriza por una mutación de la herencia autosómica dominante en la cual el FVW posee todos su multímeros; pero tiene una baja afinidad, lo que impide la unión plaquetaria $(34,35)$. Clínicamente, se ha visto que este tipo puede ser equivalente a la tipo IIB (41).

Tipo 2N. Este subtipo está dado por una mutación autosómica recesiva que puede darse en los exones $18-20$ o en el sitio de clivaje por la furina para la remoción del propéptido (8) en el sitio de unión al FVIII, por lo que genera una disminución de la afinidad del FVW al factor VIII y, por consiguiente, disminución de sus concentraciones plasmáticas. Este mecanismo fisiopatológico explica por qué este tipo puede simular el cuadro clínico de la hemofilia tipo A $(21,41,42)$.

\section{Seudo-Von Willebrand}

El término seudo-Von Willebrand se refiere a una patología dada por una mutación autosómica dominante en la que hay una mutación missense en el codón 239, que remplaza una metionina por una valina y que lleva a la formación de un receptor GPP IB hiperfuncionante (43). El cambio estructural del receptor lleva a que este se encuentre constantemente activado y, por lo tanto, se mantenga unido al FVW, por lo que simula la EVW con un FVW sin ninguna alteración (1).

\section{Cuadro clínico}

La EVW se caracteriza por cursar con sangrados frecuentes; sin embargo, usualmente médicos y pacientes desatienden este síntoma y así se pueden tardar años en obtener el diagnóstico correcto; por esto, se debe tener una alta sospecha clínica de la enfermedad si se presentan sangrados a repetición, especialmente mucocutáneos como: epistaxis, de la mucosa oral, púrpuras, petequias, sangrados gastrointestinales, y en las mujeres jóvenes en edad reproductiva, sangrados menstruales abundantes (23).

La presentación del cuadro clínico depende del tipo de enfermedad del paciente. Por lo general, las formas más severas se diagnostican en la infancia o adolescencia; mientras que las formas leves se diagnostican en pacientes adultos (44). Los signos y síntomas de la EVW más frecuentes en mujeres en edad reproductiva son (45):

La presencia de coágulos en el flujo menstrual de más de 2,5 $\mathrm{cm}$ de diámetro.

La necesidad de cambiar la toalla higiénica o el tampón cada hora o con mayor frecuencia.

Síntomas de anemia: cansancio, fatiga o disnea.

A pesar de ser una patología que presenta sangrados como síntoma principal, es necesario diferenciar entre aquellos dados por trastornos de la coagulación y otras patologías (46). Se han disen\#ado cuestionarios para dar una puntuacio\#n segu\#n los si\#ntomas, denominados bleeding score (47), como herramienta para cuantificar las manifestaciones y contribuir a discriminar entre sujetos con EVW y sin esta, y un cuestionario y score pedia\# tricos. Para definir sangrado menstrual excesivo, se utiliza el Pictorial Bleeding Assessment Chart (48). 


\section{Diagnóstico}

Hacer un correcto diagnóstico de la EVW es fundamental, por lo que es necesario basar la evaluación clínica en tres pilares: la historia personal, la historia familiar y la evaluación paraclínica.

\section{Historia personal}

Es necesario indagar específicamente por síntomas hemorrágicos como sangrados mucocutáneas excesivos, hematomas sin motivo aparente, epistaxis y laceraciones con hemorragias excesivas o de difícil control (41). En las mujeres es fundamental hacer una detallada descripción de los patrones menstruales, ya que esta puede ser la única manifestación de la enfermedad (49).

\section{Historia familiar}

Se deben evaluar los antecedentes familiares de hemorragias excesivas. Si bien es común encontrar antecedentes familiares, dejar de hallarlos no descarta la patología, ya que existen algunas presentaciones de la enfermedad que tienen un patrón hereditario autosómico recesivo y grados de penetrancia variable (50).

\section{Exámenes paraclínicos}

El tamizaje de la EVW es limitado, por la baja fiabilidad de los exámenes utilizados. El tiempo de tromboplastina parcial puede encontrarse normal en casos de EVW de tipo 1 leve o prolongado en caso de niveles bajos de FVIII, lo que restringe su uso como herramienta de cribado (51). Por otro lado, a pesar de su baja sensibilidad y especificidad, los análisis de función plaquetaria deben contemplarse en el abordaje diagnóstico de la enfermedad $(38,52,53)$.

Considerando que no se cuenta con una prueba sencilla y aislada que permita diagnosticar la EVW, debe utilizarse una batería de pruebas de laboratorio (tabla 2), cuyos hallazgos varían dependiendo del tipo de EVW que padece el individuo (tabla 3). Adicionalmente, se encuentran disponibles pruebas de laboratorio específicas que contribuyen a confirmar el tipo y subtipo de EVW (tabla 4). El gen del FVW tiene múltiples variaciones de la secuencia benigna y sus deleciones amplias no se detectan en la secuenciación habitual de ADN, lo que dificulta el diagnóstico genético (54).

\section{Tabla 2.}

Pruebas de laboratorio en el diagnóstico de la enfermedad de Von Willebrand

\begin{tabular}{|l|l|l|}
\hline Prueba & Abreviatura & Objetivo \\
\hline $\begin{array}{l}\text { Antígeno del } \\
\text { FVW }\end{array}$ & FVW:Ag & $\begin{array}{l}\text { Mide la cantidad total de proteína de } \\
\text { FVW presente. }\end{array}$ \\
\hline $\begin{array}{l}\text { Actividad del } \\
\text { FVW }\end{array}$ & FVW:RCo & $\begin{array}{l}\text { Valora la interacción entre el FVW y las } \\
\text { plaquetas mediada por la ristocetina. }\end{array}$ \\
\hline $\begin{array}{l}\text { Cociente } \\
\text { actividad/antigen } \\
\text { o FVW }\end{array}$ & FVW:RCo/FVW:Ag & $\begin{array}{l}\text { Una reducción del cociente }(<0,7) \text { se } \\
\text { encuentra en la EVW de tipos 2A, 2B y } \\
\text { 2M }\end{array}$ \\
\hline $\begin{array}{l}\text { Actividad del } \\
\text { factor VIII }\end{array}$ & FVIII & $\begin{array}{l}\text { Mide el FVIII circulante, que puede ser } \\
\text { muy bajo en la EVW de tipo 2N y 3 }\end{array}$ \\
\hline $\begin{array}{l}\text { Distribución de } \\
\text { los multímeros }\end{array}$ & Multímeros del FVW & $\begin{array}{l}\text { Permite visualizar los multímeros de la } \\
\text { EVW, empleados para identificar los } \\
\text { multimeros de alto peso molecular, que } \\
\text { faltarán en la EVW de tipos 2A y 2B }\end{array}$ \\
\hline
\end{tabular}

adaptado de (52).

Tabla 3.

Hallazgos de laboratorio esperados en cada tipo de la enfermedad de Von Willebrand

\begin{tabular}{|l|l|l|l|l|l|l|}
\hline & Tipo 1 & Tipo 3 & Tipo 2A & Tipo 2B & Tipo 2M & Tipo 2N \\
\hline FvW:Ag & $\downarrow$ & Ausente & $\downarrow$ & $\downarrow$ & $\downarrow$ & $\downarrow$ \\
\hline FvW:RCo & $\downarrow$ & Ausente & $\downarrow \downarrow$ & $\downarrow \downarrow$ & $\downarrow \downarrow$ & $\downarrow$ \\
\hline FVIII & Normal & $\downarrow \downarrow$ & $\begin{array}{l}\text { Normal o } \\
\downarrow\end{array}$ & $\begin{array}{l}\text { Normal o } \\
\downarrow\end{array}$ & $\begin{array}{l}\text { Normal o } \\
\downarrow\end{array}$ & $\downarrow \downarrow$ \\
\hline $\begin{array}{l}\text { Distribución } \\
\text { multímeros }\end{array}$ & Normal & Ausente & $\begin{array}{l}\text { Pérdida de } \\
\text { MAPM }\end{array}$ & $\begin{array}{l}\text { Pérdida de } \\
\text { MAPM }\end{array}$ & Normal & Normal \\
\hline
\end{tabular}

FvW:Agantígeno del FVW

FvW:RCoactividad del cofactor de ristocetina del FVW FVIIIfactor VIII

MAPMmultímeros de alto peso molecular adaptado de (52) 
Tabla 4.

Pruebas de laboratorio especializadas en la enfermedad de Von Willebrand

\begin{tabular}{|c|c|c|c|c|c|c|c|c|c|}
\hline & \begin{tabular}{|l|} 
Normal \\
\end{tabular} & \begin{tabular}{|l|l} 
Tipo 1 \\
\end{tabular} & \begin{tabular}{|l}
$\begin{array}{l}\text { Tipo } \\
\text { 1C }\end{array}$ \\
\end{tabular} & Tipo 3 & Tipo 2A & \begin{tabular}{|l|l|} 
Tipo 2 \\
\end{tabular} & $\begin{array}{l}\begin{array}{l}\text { Tipo } \\
2 \mathrm{~N}\end{array} \\
\end{array}$ & $\begin{array}{l}\text { Tipo } \\
2 \mathrm{M}\end{array}$ & \begin{tabular}{|l|l|} 
EVW-PT \\
\end{tabular} \\
\hline FVW:Ag & $\mathrm{N}$ & $\downarrow$ & $\downarrow$ & Ausente & $\downarrow$ & $\downarrow$ & No & No & $\Downarrow$ \\
\hline FVW:RCo & $\mathrm{N}$ & $\downarrow$ & $\Downarrow$ & Ausente & $\$ 4 t$ & $\Downarrow$ & 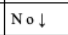 & 4 & $\downarrow$ \\
\hline FVIII: C & $\mathrm{N}$ & Nol & $\downarrow$ & $\begin{array}{l}2-10 \\
\text { U//dL }\end{array}$ & Nol & 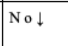 & 4 & $\mathrm{~N}$ & Nol \\
\hline $\begin{array}{l}\text { Cociente } \\
\text { FVWpp/FV } \\
\text { W:Ag }\end{array}$ & $\mathrm{N}$ & $\mathrm{N}$ & $\uparrow \uparrow$ & Ausente & No $\uparrow$ & $\uparrow$ & $\mathrm{N}$ & $\mathrm{N}$ & $\uparrow$ \\
\hline RIPA & $\mathrm{N}$ & \begin{tabular}{|l|l} 
A \\
menudo \\
N
\end{tabular} & $\downarrow$ & Ausente & $\downarrow$ & \begin{tabular}{|l|} 
A \\
menudo \\
N
\end{tabular} & N & Nol & \begin{tabular}{|l|l|} 
A \\
menudo \\
$\mathrm{N}$
\end{tabular} \\
\hline LD-RIPA & Ausente & Ausente & Ausente & Ausente & Ausente & $\pi \uparrow \uparrow$ & \begin{tabular}{|l|l} 
Ausente \\
\end{tabular} & \begin{tabular}{|l} 
Ausente \\
\end{tabular} & $\pi \uparrow \uparrow$ \\
\hline PFA & $\mathrm{N}$ & No 1 & $\uparrow$ & $\uparrow \uparrow \uparrow$ & $\uparrow$ & $\uparrow$ & N & $\uparrow$ & $\uparrow$ \\
\hline TH & N & No $\uparrow$ & $\uparrow$ & $\Uparrow \uparrow \uparrow$ & $\uparrow$ & $\uparrow$ & N & $\uparrow$ & $\uparrow$ \\
\hline $\begin{array}{l}\text { Recuento } \\
\text { plaquetario }\end{array}$ & $\mathrm{N}$ & $\mathrm{N}$ & $\mathrm{N}$ & $\mathrm{N}$ & $\mathrm{N}$ & Nol & N & N & $\downarrow$ \\
\hline $\begin{array}{l}\text { Multimeros } \\
\text { del FVW }\end{array}$ & $\mathrm{N}$ & N pero $\downarrow$ & \begin{tabular}{l|} 
N pero \\
$\downarrow$
\end{tabular} & Ausente & \begin{tabular}{|l|} 
Anorma \\
1
\end{tabular} & \begin{tabular}{|l|l} 
Anorma \\
1
\end{tabular} & $\begin{array}{l}\text { N pero } \\
\downarrow\end{array}$ & N pero & Anormal \\
\hline
\end{tabular}

FVW:Agantígeno del FVW

FVW:RCoactividad del FVW por el cofactor de ristocetina

FVIII:Cactividad coagulante del factor VIII

FVWpppropéptido del FVW

RIPAagregación plaquetaria

inducida por ristocetina

LD-RIPAagregación plaquetaria

inducida con dosis bajas de ristocetina

THtiempo de hemorragia

EVW-TPenfermedad de Von

Willebrand de tipo plaquetario Nnormal

$\mathrm{N}$ pero \#normal pero de intensidad reducida adaptado de (52)

Durante la valoración paraclínica inicial del paciente con sospecha de EVW se tiene como objetivo detectar la presencia o ausencia del FVW (FVW:Ag), sus productos en la cascada de coagulación (FVIII:C) y su capacidad de unión o no a GPIba mediado por ristocetina (FVW:RCo). Por otro lado, las pruebas de segunda línea incluyen el análisis del patrón multimérico de la proteína, que brinda información acerca de su estructura como es el caso de la EVW tipo 2A y 2B (tabla 2) y el análisis de RIPA (prueba de agregación plaquetaria inducida por ristocetina entre 0,7 y $1,2 \mathrm{mg} / \mathrm{ml}$ ), que detecta alteraciones en la respuesta a la ristocetina, como en la EVW $2 \mathrm{~A}$ y $2 \mathrm{M}(55)$.

Se encuentran disponibles otras pruebas especializadas como LD-RIPA $(<0,7 \mathrm{mg} / \mathrm{ml})$, que evalúa la respuesta de agregación plaquetaria a bajas dosis de ristocetina, provocando su aglutinación como en el caso de la EVW tipo
2B y el cociente entre la determinación inmune del propéptido (FVWpp) y FVW:Ag (FVWpp/ FVW:Ag), que constituye un marcador de depuración acelerada del FVW (56). Cuando se evidencia un aumento $>2-3$ en dicho cociente, es utilizado para distinguir una manifestación severa de EVW tipo 1 de la EVW tipo 3 (53). En la figura 2 se resume en un algoritmo las indicaciones para el diagnóstico de la EVW y sus subtipos $(57,58)$.

Figura 2.

Algoritmo diagnóstico para la enfermedad de Von Willebrand y sus subtipos

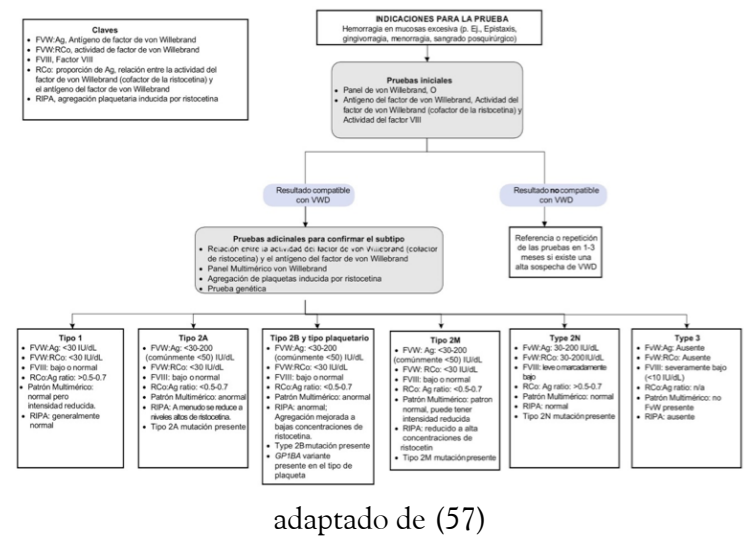

Las pruebas iniciales en pacientes con sospecha de trastornos hemorrágicos suelen incluir el hemograma, recuento de plaquetas, tiempo de protrombina (PT) y tiempo parcial de tromboplastina activada (aPTT). Sin embargo, tienen un uso limitado en el diagnóstico de EVW porque son normales en la mayoría de los subtipos, no son sensibles, ni específicas para la EVW. Si el paciente no tiene anomalías detectadas por las pruebas de hemostasia iniciales se recomienda utilizar el algoritmo indicado.

\section{Tratamiento}

En pacientes con EVW, el objetivo terapéutico es corregir el defecto dual de la hemostasia. Las opciones terapéuticas incluyen el uso de desmopresina, que libera FVW endógeno de las células endoteliales y el FVW exógeno de los concentrados derivados de plasma de FVW/ FVIII. Esto logra incrementar los niveles de 
FVW, por lo que previenen eventos hemorrágicos severos (59).

Desmopresina (1-ácido8-D-arginina vasopresina) (DDAVP). Es un derivado sintético de la hormona antidiurética que aumenta las concentraciones plasmáticas de FVW y FVIII. Se cree que este efecto se da por la activación del endotelio vascular, que libera el FVW desde sus zonas de almacenamiento y mejora los niveles de FVIII al evitar su degradación (60). El FVW y FVIII aumentan de tres a cinco veces por encima de los niveles basales cuando se inyecta DDAVP por vía intravenosa (IV), subcutánea (SC) o intranasal (61). La dosis IV es de 0,3-0,4 $\mu \mathrm{g} / \mathrm{kg}$, máximo $20 \mu \mathrm{g}$. Se infunde diluida en 50 a $100 \mathrm{ml}$ de solución de $\mathrm{NaCl}$ al 0,9\% en 20 a 30 min. Por vía intranasal la dosis recomendada es de 150 a $300 \mu \mathrm{g}(62)$.

Los pacientes con EVW tipo 1 presentan mejor respuesta al uso de DDAVP. En la EVW tipo 2 la respuesta se correlaciona con el tipo de defecto congénito: para el subtipo $2 \mathrm{~A}$ se necesita la prueba terapéutica de DDAVP, porque habrá liberación de FVW con multímeros de alto peso molecular disfuncionales. En la variante 2B se encuentra contraindicada, por trombocitopenia secundaria a la agregación plaquetaria. En la $2 \mathrm{M}$ se espera una respuesta escasa por alteración en el receptor de unión a la plaqueta. En el subtipo $2 \mathrm{~N}$ ocurre un incremento transitorio del FVIII, al no poder ser estabilizado por el FVW, por encontrarse mutado el sitio de unión. En la EVW tipo 3 no hay producción endógena de factores, por lo que el uso de DDAVP no genera efecto terapéutico (37).

Debe utilizarse cuando los pacientes van a someterse a procedimientos con riesgo de sangrado leve a moderado. Adicionalmente, se encuentra indicado en pacientes con sangrado menstrual excesivo o epistaxis. Deben tenerse en cuenta los posibles efectos adversos como la cefalea, el enrojecimiento facial y la hiponatremia, que debe prevenirse evitando la ingesta de líquidos posterior a la aplicación. No se contempla su uso en menores de dos años (23).

Concentrados de FVW/FVIII. Los concentrados derivados del plasma están indicados en pacientes que no responden a la desmopresina, que requieren hemostasia mayor o mantener el efecto hemostático por al menos 2-3 días. Los productos que contienen FVW inicialmente se prescribieron para el tratamiento de la hemofilia, y la cantidad de FVW dependía, en gran medida, del proceso de purificación de cada producto (63). Existen concentrados puros de FVW que aumentan inmediatamente los niveles de esta molécula sin incremento inmediato del FVIII; se prefieren estos para procedimientos programados. Para los sangrados severos o intervenciones de urgencia es necesario aplicar concentrados duales que contienen ambos factores. Es necesario familiarizarse con los diferentes coeficientes de los productos disponibles y basar la elección en los niveles basales del paciente (23). La eleccio\#n del compuesto se basa en su cantidad de FVW y de FVIII. En situaciones de hemorragia severa se prefieren agentes con mayor cantidad de FVIII seguidos de mantenimiento con compuestos con alta cantidad de FVW (preparados recombinantes de FVW) (63).

Hay varios productos disponibles que contienen FVW en alta concentración (64):

Concentrados de FVIII de "pureza intermedia": Humate-P®.

Concentrados de FVW más altamente purificados; se pasteurizan o se tratan con detergentes solventes para reducir el riesgo de transmisión de virus:

Alphanate ${ }^{\circledR}$

Fandhi ${ }^{\circledR}$

Wilfactin ${ }^{\circledR}$

FVW humano recombinante, que contiene un espectro completo de multímeros de FVW: Vonvendi®.

La dosis y la frecuencia de administración se deciden teniendo en cuenta el peso del paciente, el tipo y la gravedad de los sangrados o intervención quirúrgica y la función de control de los parámetros clínicos y analíticos pertinentes (65). En general, el objetivo es mantener la actividad del FVIII y el FVW (medido como actividad del cofactor de ristocetina [FVW:RCo]) entre el 50 y el $100 \%$ para hemorragias graves o cirugía mayor. La infusión de 20-50 unidades internacionales (UI) $/ \mathrm{kg}$ de 
actividad del cofactor de ristocetina eleva la concentración plasmática a 50 y a $100 \%$, o 0,7 unidades $/ \mathrm{ml}(70 \%)$; se administra cada 12 a 24 horas. Una dosis de carga de 50 a $80 \mathrm{UI} / \mathrm{kg}$ se administra al comienzo del tratamiento para asegurar que se alcance el nivel deseado (66).

Adicionalmente, deben tenerse en cuenta las recomendaciones para procedimientos quirúrgicos: cirugi\#a menor, niveles de FVW:RCo $\geq 50 \mathrm{UI} / \mathrm{dL}$ el di\# a del procedimiento y el primer di\# a postoperatorio, y $>30 \mathrm{UI} / \mathrm{dL} 2$ a 5 di\# as postoperatorio, o hasta la cai\#da de la escara. Cirugi\# a mayor, los niveles de FVW:RCo deben ser $100 \mathrm{UI} / \mathrm{dL}$ el di\# a del procedimiento y durante el primer di\# a postoperatorio, y deben mantenerse $\geq 50 \mathrm{UI} / \mathrm{dL}$ de 7 a 14 di\# as o hasta la cicatrizacio\#n completa (67).

\section{Profilaxis}

Actualmente, no existe consenso de las indicaciones para dar profilaxis a los pacientes con EVW. Se han desarrollado múltiples estudios en su mayoría en el marco de The Von Willebrand Disease Prophylaxis Network (68). Los estudios realizados han demostrado que la administración de concentrado de FVWristocetina en dosis que varían entre 39 a $60 \mathrm{U}$ FVW:Rco/kg a largo plazo logra reducir eventos hemorrágicos (epistaxis, hemartrosis, sangrados gastrointestinales) y mejora la calidad de vida, disminuyendo el tiempo de estancia hospitalaria y el ausentismo escolar (69).

\section{Terapias coadyuvantes}

Algunas situaciones ameritan adicionar agentes antifibrinolíticos como el ácido tranexámico o el ácido épsilon aminocaproico. Están indicados como terapia coadyuvante en situaciones de hemorragia leve-moderada, procedimientos dentales, sangrado menstrual excesivo y epistaxis (70).

Ambos medicamentos son a\#cidos gammaamino-carboxi\#licos ana\#logos de la lisina; actúan inhibiendo la conversión de plasminógeno a plasmina, que actúa degradando la fibrina, por lo que previene la formación de la red de fibrina y la estabilización del coa\#gulo (71). El a\#cido epsilo\# $\mathrm{n}$ aminocaproico se usa a una dosis inicial de 100 a $150 \mathrm{mg} / \mathrm{kg}$, seguido de una infusio\#n de 10 a $15 \mathrm{mg} / \mathrm{kg} / \mathrm{h}$, el a\#cido tranexa\# mico es 7 a 10 veces ma\#s potente, por lo que puede usarse a bajas dosis; dosis inicial de $10 \mathrm{mg} / \mathrm{kg}$ seguido de $1 \mathrm{mg} / \mathrm{kg} / \mathrm{hora}$ (46).

En mujeres con sangrado menstrual excesivo, la administración de terapia hormonal combinada funciona parcialmente, al elevar las concentraciones de FVW y FVIII; el uso de sistemas intrauterinos con progesterona, frecuentemente, aportan beneficios clínicos considerables $(1,72)$.

\section{Discusión}

La EVW es el trastorno hematológico más comúnmente heredado, con un patrón de herencia autosómica que afecta al $1 \%$ de la población total. En su mayoría, cursa asintomático y existen variantes graves que pueden comprometer la vida. Para el 2018, en Colombia se documentaron 1504 pacientes con esta patología, con una prevalencia de 3 casos por 100.000 habitantes, clasificándose como una enfermedad huérfana según los lineamientos de nuestro país (16).

Las frecuentes discrepancias entre los resultados de laboratorio, función in vivo y cuadro clínico plantean un reto diagnóstico para el médico, quien debe sospechar esta condición en pacientes con hemorragias frecuentes.

En los últimos años, la caracterización de la estructura del FVW y su producción molecular ha influenciado el diagnóstico paraclínico y el abordaje diagnóstico (73). El entendimiento de los mecanismos biológicos subyacentes aporta a la creación de terapias que mejoran los desenlaces y la calidad de vida de los pacientes.

Asimismo, la optimización del tratamiento a largo plazo de estos pacientes demanda la conducción de ensayos clínicos, con el objetivo de ofrecer mejor evidencia que permita tomar decisiones clínicas acertadas. Por otro lado, los avances en ingeniería genética resultan 
prometedores para proveer una terapia genética dirigida a pacientes con EVW (67).

Mientras avanzan las investigaciones es necesario promover la implementación de estrategias de salud pu\#blica que mejoren el acceso a la salud ligado a buenas pra\#cticas cli\# nicas, basadas en el trabajo coordinado de un equipo multidisciplinario con los conocimientos y las bases necesarias para brindar la mejor atención posible a nuestros pacientes. Si bien en Colombia existe un lineamiento que precisa los contenidos mínimos para la conformación de programas para la atención integral de pacientes con hemofilia y otros defectos de la coagulación (74), es frecuente el subdiagnóstico de estas condiciones, persiste una brecha importante por disminuir y solo un porcentaje de los pacientes recibe tratamiento en nuestro país. Es prioritario revisar los resultados de las estrategias y acciones movilizadas por los gobiernos de otras naciones de América Latina y el mundo, con el objetivo de implementar las mejores estrategias en nuestro país.

Es fundamental evitar la fragmentación de la atención por medio de los programas de atención integral y supervisión permanente en los pacientes con EVW en aras de reducir la carga de enfermedad producida por esta condición, evitar los desenlaces adversos en salud, mejorar la calidad de vida de los pacientes y optimizar el uso de recursos en el sistema de salud colombiano (74).

La presente revisión documentó la información más relevante y actualizada encontrada en la literatura con el objetivo de llenar los vacíos encontrados en la práctica clínica diaria en nuestro país.

\section{Agradecimientos}

A Natalia García, estudiante de medicina por la elaboración de la adaptación de la figura 1.

\section{Referencias}

$\begin{array}{lrrr}\text { 1. Espitia-de } & \mathrm{La} & \mathrm{Hoz} & \mathrm{FJ} . \\ \text { A } & \text { qualitative } & \text { review of the }\end{array}$

literature regarding managing Von Willebrand disease in obstetrics and gynaecology. Rev Colomb Obstet Ginecol [Internet]. 2010;61(2):151-9. Disponible en: https://revista.fecolsog. org/index.php/rcog/article/view/284

2. Bustamante G. Enfermedad de Von Willebrand. Rev Actual Clínica. 2011;4:169-72.

3. Margaret E Rick M. Clinical presentation and diagnosis of von Willebrand disease. UpToDate. 2018.

4. Instituto Nacional de Salud. Boletín epidemiológico 2019. Bogotá; 2019.

5. Lozano ML, Navarro$\mathrm{Nez}$ L, Martínez C, Rivera J. Alteraciones de las plaquetas. Etiopatogenia, clasificación, manifestaciones clínicas, diagnóstico y actitudes terapéuticas. Medicine (Baltimore). 2009;10(22):1465-74.

6. Robertson J, Lillicrap D, James PD. Von Willebrand Disease. Pediatr Clin North Am. 2008;55(2):377-92.

7. Valle D, Beaudet AL, Vogelstein

B, Kinzler K, Antonarakis Stylianos E, Ballabio A, et al. The online metabolic and molecular bases of inherited disease. New York: McGraw Hill; 2011.

8. Meschengieser SS. Enfoque diagnóstico de la enfermedad de von Willebrand y hemofilia adquirida en nuestro país. Hematologia. 2015;19(tipo 1):25-31.

9. National Heart Lung and Blood Institute. The diagnosis, evaluation, and management of von willebrand disease. New York; 2007.

10. Woods A, Blanco A, Kempfer A, Paiva J, Bermejo E. Factor Von Willebrand y enfermedad de Von Willebrand: nuevos enfoques diagnósticos. Acta Bioquím Clín Latinoam. 2016;50(2):273-89. 
11. SDS. Diagnóstico y tratamiento de la enfermedad de Von Willebrand: guía de práctica clínica. México; 2010. 12. Rodeghiero F, Castaman G, Dini E. Epidemiological investigation of the prevalence of von Willebrand's disease. Blood. 1987;69(2):454-9.

13. Centro Nacional de Excelencia Tecnología en Salud. Guía de práctica clínica diagnóstico y tratamiento de la enfermedad de Von Willebrand. Ciudad de México; 2012.

14. Echahdi $\mathrm{H}$, El Hasbaoui B, El Khorassani M, Agadr A, Khattab M. Von Willebrand's disease: case report and review of literature. Pan Afr Med J. 2017;27:1-7.

15. Cuenta de Alto Costo. Situación de la hemofilia en Colombia 2016. Bogotá; 2016.

16. Cuenta de Alto Costo. Situación de la hemofilia en Colombia 2018. Bogotá; 2018.

17. Kessler CM, Makris M. Von Willebrand's disease: a report from a meeting in the Aland Islands. Haemophilia. 2016;18(September 2010):22-5.

18. Baronciani L, Goodeve A, Peyvandi F. Molecular diagnosis of von Willebrand disease. Haemophilia. 2017;23(2):188-97.

19. Peters RT, Leksa NC, Lu Q, Dong X, Knockenhauer KE, Arndt JW, et al. The von Willebrand factor D'D3 assembly and structural principles for factor VIII binding and concatemer biogenesis. Blood. 2019;268:blood-2018-10-876300.

20. Lillicrap D. Von Willebrand disease-Phenotype versus genotype: deficiency versus disease. Thromb Res. 2007;120.

21. James PD, Goodeve AC. Von Willebrand Disease. Genet Med.
2013;13(5):365-76. https://doi.org/10. 1097/GIM.0b13e3182035931.

22. Goodeve AC. The genetic basis of von Willebrand disease. Blood Rev. 2010;24(3):123-34.

23. Ng CJ, Di Paola J. Von Willebrand disease: diagnostic strategies and treatment options. Pediatr Clin North Am. 2018;65(3):527-41.

24. Zheng XL. ADAMTS13 and von Willebrand Factor in thrombotic thrombocytopenic purpura. Annu Rev Med. 2015;66(1):211-25.

25. Schneppenheim R. The pathophysiology of von Willebrand disease: therapeutic implications. Thromb Res. 2011;128(suppl. 1):S3-7.

26. Paola J Di, Montgomery RR, Gill JC, Flood V. Hemophilia and von Willebrand disease. En: Nathan and Oski's hematology and oncology of infancy and childhood. Philadelhia: Sounders; 2014. p. 1028-54.

27. Swami A, Kaur V. Von Willebrand disease: a concise review and update for the practicing physician. Clin Appl Thromb. 2017;23(8):900-10.

28. Foot J, Schneppenheim R, Lenting PJ, Aponte-Santamaría C, Kolšek K, Gräter F, et al. Structure and dynamics of the platelet integrin-binding $\mathrm{C} 4$ domain of von Willebrand factor. Blood. 2019;133(4):366-76.

29. Rodeghiero F. Von Willebrand disease: pathogenesis and management. Thromb Res. 2013;131(suppl.1):S47-50.

30. James P, Rydz N. Structure, biology, and genetics of Von Willebrand factor [Internet]. En: Hematology. 7th ed. Elsevier; 2018. p. 2051-63. Disponible en: http://inkinghub.elsevier.com/retri eve/pii/B9780323357623001384

31. Lillicrap D. Von Willebrand disease: advances in pathogenetic 
understanding, diagnosis, and therapy. Blood. 2013;122(23):254-60.

32. Bury L, Malara A, Momi S, Petito E, Balduini A, Gresele P. Mechanisms of thrombocytopenia in platelet-type Von Willebrand disease. Haematologica. 2019;104(7):1473-81. https://doi.org/1 0.3324/haematol.2018.200378.

33. Huddleston LL, Liu LL. Hemostasis. En: Basics of anesthesia. 7th Ed. Elsevier; 2018. p. 377-94.

34. Sadler JE, Budde U, Eikenboom JCJ, Favaloro EJ, Hill FGH, Holmberg L, et al. Update on the pathophysiology and classification of von Willebrand disease: A report of the Subcommittee on von Willebrand factor. J Thromb Haemost. 2006;4(10):2103-14.

35. Favaloro EJ, Pasalic L, Curnow J. Laboratory tests used to help diagnose von Willebrand disease: an update. Pathology. 2016;48(4):303-18.

36. Eikenboom JCJ. Congenital von Willebrand disease type 3: Clinical manifestations, pathophysiology and molecular biology. Best Pract Res Clin Haematol. 2001;14(2):365-79.

37. Leebeek FWG, Eikenboom JCJ. Von Willebrand's Disease. N Engl J Med. 2016;27(2):2067-80.

38. Roberts JC, Flood VH. Laboratory diagnosis of von Willebrand disease. Int J Lab Hematol. 2015;37(S1):11-7.

39. Springer TA. von Willebrand factor, Jedi knight of the bloodstream Timothy. Blood. 2014;124(9):1412-26.

40. Federici AB, Mannucci PM, Castaman G, Baronciani L, Bucciarelli P, Canciani MT, et al. Clinical and molecular predictors of thrombocytopenia and risk of bleeding in patients with von Willebrand disease type 2B: a cohort study of 67 patients. Blood [Internet]. 2009 Jan 15 [citado 2019 Jul 9];113(3):526-34. Disponible en: http://www.ncbi.nlm.nih.gov/pubm ed/18805962

41. James P, Lillicrap D. Enfermedad de Von Willebrand: introducción para médicos de atención primaria. Monteal: Federación Mundial de Hemofilia. 2009. Disponible en: http://www1.wfh.org/publication/fi les/pdf-1205.pdf

42. Fressinaud E. Orphanet: enfermedad de Von Willebrand tipo 2N [Internet]. 2009 [citado 2019 jul 9]. Disponible en: https://www.orpha.net/consor/cgibin/OC_Exp.php?lng $=$ es\&Expert $=16$ 6093.

43. Russell SD, Roth GJ. Pseudovon Willebrand disease: a mutation in the platelet glycoprotein iba gene associated with a hyperactive surface receptor. Blood [Internet]. 1993 [citado 2019 Jul 9];81(7):1787-91. Disponible en: https://www.bloodjourn al.org

44. Tosetto A, Rodeghiero F, Castaman G, Goodeve A, Federici AB, Batlle J, et al. A quantitative analysis of bleeding symptoms in type 1 von Willebrand disease: Results from a multicenter European study (MCMDM-1 VWD). J Thromb Haemost. 2006;4(4):766-73.

45. Rick ME. Clinical presentation and diagnosis of von Willebrand disease. UpToDate [Internet]. 2019 [citado 2019 Jul 9]. Disponible en: https://www -uptodate-com.ezproxy.javeriana.edu. $\mathrm{co} /$ contents/clinical-presentation-anddiagnosis-of-von-willebrand-disease?se arch $=$ Clinicalpresentationanddiagnos isofvonWillebranddisease $\&$ source $=$ se arch_result\&selectedTitle $=1 \sim 150 \& u$ sage_type

46. Williams $M$, Patel J. Von Willebrand disease: diagnosis and management. Paediatr Child Heal (United Kingdom). 2015;25(8):354-9. 
47. Bowman M, James P. Bleeding scores for the diagnosis of von Willebrand disease. Semin Thromb Hemost [Internet]. 2017 Jul 14 [citado 2019 Jul 9];43(05):530-9. Disponible en: http://www.thieme-connect.de/D OI/DOI?10.1055/s-0036-1597289

48. Janssen C, Scholten P, Heintz A. A simple visual assessment technique to discriminate between menorrhagia and normal menstrual blood loss. Obstet Gynecol [Internet]. 1995 Jun [citado 2019 Jul 9];85(6):977-82. Disponible en: http://www.ncbi.nlm.nih.gov/pubm ed/7770270.

49. Kujovich JL. Von Willebrand's disease and menorrhagia: Prevalence, diagnosis, and management. Am J Hematol. 2015;79(3):220-8.

50. Swystun LL, James PD. Genetic diagnosis in hemophilia and von Willebrand disease. Blood Rev. 2017;31(1):47-56.

51. Miller JL, Koneti RA. Platelet disorders and von Willebrand disease. In: Henry's clinical diagnosis and management by laboratory methods. Philadelphia: Elsevier-Saunders; 2016. p. 812-33.

52. Flood VH, Scott JP. Enfermedad de Von Willebrand. En: Nelson, tratado de pediatría. 17th ed. Philadelphia: Elsevier Science; 2011. p. 169-72.

53. Goldman L, Schafer AI, Nichols WL. Enfermedad de Von Willebrand y anomalías hemorrágicas de las plaquetas y de la función vascular. En: Tratado de medicina interna [Internet]. 4th ed. 2013. p. 1135-40. Disponible en: http://www.sciencedirect.com/scie nce/article/pii/B978848086971300176 5

54. Goodeve A. Diagnosing von Willebrand disease: Genetic analysis. Hematology. 2016;2016(1):678-82.
55. Blanco A. Enfermedad de von Willebrand: novedades en diagnóstico de laboratorio. Hematologia [Internet]. 2018 [citado 2019 Jul 9];22:54-64. Disponible en: http://www.sah.org.ar/Revista/num eros/vol22/sup/12_Enfermedad_de_vo n_Willebrand_Novedades_diagnostic o_de_laboratorio.pdf.

56. Stufano F, Baronciani L, Peyvandi F. Diagnóstico de la enfermedad de Von Willebrand: caracterización fenotípica [Internet]. Montreal: Federación Mundial de la Hemofilia; 2017 [cited 2019 Jul 9]. Disponible en: http://www1.wfh.org/pu blications/files/pdf-1694.pdf

57. ARUP Consult. Von Willebrand Disease - VWD [Internet]. 2019 [citado 2019 Jul 9]. Disponible en: https://arupconsult.com/content/v on-willebrand-disease.

58. Favaloro EJ, Bodó I, Israels SJ, Brown SA. Von Willebrand disease and platelet disorders. Haemophilia [Internet]. 2014 May [citado 2019 Jul 9];20:59-64. Disponible en: http://www.ncbi.nlm.nih.gov/pubm ed/24762277.

59. Lavin M, O'Donnell JS. How I treat low von Willebrand factor levels. Blood. 2019;133(1):795-804.

60. Leissinger C, Carcao M, Gill JC, Journeycake J, Singleton T, Valentino L. Desmopressin (DDAVP) in the management of patients with congenital bleeding disorders. Haemophilia [Internet]. 2014 Mar [cited 2019 Jul 9];20(2):158-67. Disponible en: http://www.ncbi.nlm.ni h.gov/pubmed/23937614

61. Federici AB. The use of desmopressin in von Willebrand disease: The experience of the first 30 years (1977-2007). Haemophilia [Internet]. 2007 Dec 21 [cited 2019 Jul 
9];14:5-14. Disponible en: http://www. ncbi.nlm.nih.gov/pubmed/18173689.

62. Soto V, Morales MM, Verdugo P. Empleo del DDAVP en el manejo de la enfermedad de Von Willebrand. Rev Chil Pediatr [Internet]. 2005 Apr [citado 2019 Jul 9];76(2):193-7. Disponible en: http://www.scielo.cl/scielo.php?scri pt $=$ sci_arttext $\&$ pid $=$ S0370-4106200 $5000200011 \& \operatorname{lng}=$ en\&nrm $=$ iso\&th $\mathrm{g}=\mathrm{en}$

63. Martínez-Murillo C. Enfermedad de von Willebrand: el reto en el diagnóstico y el tratamiento. Rev Hematol Mex [Internet]. 2018 [citado 2019 Jul 9];19(2):61-72. Disponible en: https://www.medigraphic.com/pdfs /hematologia/re-2018/re182b.pdf

64. Genetic and Rare Diseases Information Center. Von Willebrand disease [Internet]. 2018 [citado 2019 Jul 9]. Disponible en: https://rarediseases.info.nih.gov/dis eases/7867/von-willebrand-disease.

65. Hemophilia of Georgia. Protocolos para el tratamiento de la hemofilia y de la enfermedad Von Willebrand [Internet]. Montreal: Federación Mundial de la Hemofilia; 2008 [citado 2019 Jul 9]. Disponible en: http://www1.wfh.org/publication/fi les/pdf-1138.pdf

66. Laffan MA, Lester W, O'Donnell JS, Will A, Tait RC, Goodeve A, et al. The diagnosis and management of von Willebrand disease: a United Kingdom Haemophilia Centre Doctors Organization guideline approved by the British Committee for Standards in Haematology. $\mathrm{Br} \mathrm{J}$ Haematol [Internet]. 2014 Nov [citado 2019 Jul 9];167(4):453-65. Disponible en: http://www.ncbi.nlm.nih.gov/pubm ed/25113304.

67. Lavin M, O'Donnell JS. New treatment approaches to von Willebrand disease. Hematology. 2016;2016(1):683-9.

68. Berntorp E, Abshire T, vWD PN Steering Committee. The Von Willebrand Disease Prophylaxis Network (vWD PN): Exploring a treatment concept. Thromb Res [Internet]. 2006 Jan [citado 2019 Jul 9];118:S19-22. Disponible en: http://www.ncbi.nlm.nih.gov/pubm ed/16545435

69. Abshire TC, Federici AB, Alvárez MT, Bowen J, Carcao MD, Cox Gill $\mathrm{J}$, et al. Prophylaxis in severe forms of von Willebrand's disease: results from the von Willebrand Disease Prophylaxis Network (VWD PN). Haemophilia. 2013 [citado 2019 Jul 9];19(1):76-81. https://doi.org/10.111 1/j.1365-2516.2012.02916.x

70. Manuccio Manucci P, Bonomi AB. La desmopresina (Ddavp) en el tratamiento de los trastornos de la coagulación [internet]. Montreal: Federación Mundial de la Hemofilia; 2012. Disponible en: http://www1.wfh. org/publications/files/pdf-1132.pdf

71. Cano-Franco MA, Ortiz-Orrego GE, González-Ariza SE. Dental care for coagulation hereditary disorder patients. CES Odontol. 2017;30(1):30.

72. Cuenta de Alto Costo. Coagulopatías en mujeres. Boletín de Información Técnica Especializada [Internet]. 2017;3(9). Disponible en: https://cuentadealtocosto.org/site/i mages/Publicaciones/boletines/2017/C AC_CO_2017 04_17_BOLTEC_V3 N9_dm-hemofilia_V_0_A2.pdf.

73. Buga-Corbu I, Arion C. Current therapy in children and adolescents with von Willebrand disease. J Med Life [Internet]. 2014/06/25. 2014 Jun 15;7(2):264-9. Disponible en: https://www.ncbi.nlm.nih.gov/pub $\operatorname{med} / 25408737$. 
74. Escobar G, Linares A, Solano Trujillo MH, Robledo Riaga S, Acuña Merchán L, et al. Contenidos mínimos para la conformación de programas para la atención integral de pacientes con diagnóstico de hemofilia y otros defectos de la coagulación (documento técnico) [Internet]. Bogotá: Ministerio de Salud y Protección Social, ACHO, ACHOP, Colhemofílicos y Cuenta de Alto Costo del Fondo Colombiano de Enfermedades de Alto Costo; 2017 [citado 1 oct 2019]. Disponible en: https://cuentadealtocosto.org/site/i mages/Publicaciones/2018/Contenidos _minimos_para_programas_atencion_ hemofilia_y_coagulacion.pdf.

\section{Notas}

Conflictos de interés: los autores declaran no tener conflictos de interés. 